\title{
Demulsification of Liquid Membrane Emulsion using High Voltage Glass Coated Electrode in Batch System
}

\author{
N. Othman ${ }^{1 *}$, A. Ahmad \& M. A. Piramali ${ }^{3}$ \\ 1,28z: Department of Chemical Engineering, Faculty of Chemical and Natural Resources Engineering, \\ Universiti Teknologi Malaysia, 81310 UTM Slkudai, Johor, Malaysia
}

\begin{abstract}
Demulsification is one of the key processes in emulsion liquid membrane application. This study involved the elfect of electrical field on demulsification of water in oil using batch high voltage demulsifier system. This technique widely used because of its advantages of easy manipulation of applied field direction and strength, offers cost-effective separation and minimal environmental impact combined with mechanical simplicity. Influence of various values of frequency (400-1500 Hz) and voltage was studied experimentally using Alternate Current (AC) High Voltage Demulsifier with insulated electrode. The emulsion consists of kerosene as organic phase, sulfuric acid as internal phase and span $80(3$ and $5 \mathrm{w} / \mathrm{v} \%)$ as a surfactant. The effect of emulsion preparation such as homogenizer speed, internal phase and surfactant concentrations on the stability of water-in-oil emulsion was also investigated. The results showed that the attractive forces between the water droplets under an electrical fieid increase by raising the applied electrical field strength. Meanwhile, the time required for the emulsion to separate and coalescence under electrical field increased when the stability of emulsion increased.
\end{abstract}

Keywords: Water-in-oil emulsion, demulsification, coated electrode, AC high voltage electrical field, batch system

\subsection{INTRODUCTION}

Emulsion is a heterogeneous mixture of two immiscible liquids that one of them dispersed in other phase to form globules or droplets. There are two types of emulsion such as water-in-oil $(\mathrm{W} / \mathrm{O})$ and oil-in-water $(\mathrm{O} / \mathrm{W})$ emulsions. This study only involves water-oil emulsions because it is the most encountered type of emulsion in industries especially in petroleum industry and also in purification and recovery process using emulsion liquid membrane system [1].

There are many different ways of preparing emulsions of two liquids that are insoluble. Emulsification is usually achieved by the application of mechanical energy. Initially, the

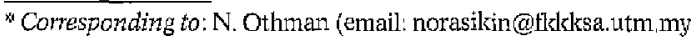

interface between the two phases is deformed to such an extent that large droplets are formed, and these large droplets are subsequently broken up into smaller ones. During emulsification, the interfacial area between two liquids increases then minimizes the surface area of liquids. Mechanical energy is required to form the emulsion.

Demulsification is one of the key processes in emulsion liquid membrane application which is mainly used for removing water and salts from crude oil in oil industry, and for the recovering the membrane phase inciuding organic solvent, surfactant, extractant, and valuable solute ions in waste treatment processes. Two principle approaches for the demulsification of loaded emulsion are chemical and physical treatments. Chemical treatment involves the addition of a demulsifier to the emulsion system [2]. Physical treatment methods include heating, centrifugation, 
$\mathrm{pH}$ adjustment, filtration, membrane separation, and electrostatic demulsification. The $\mathrm{pH}$ effect can be utilised to separate oil-in-water emulsions, but it is usually not effective in breaking water-in-oil emulsions. Centrifugation, an effective method for some emulsions, has high operating cost. Although heat treatment can reduce the viscosity of the oil phase, but this method is more expensive.

Electrostatic demulsification is a technique widely used because of its advantages of easy manipulation of applied field direction and strength. This method also offers cost-effective separation and minimal environmental impact combined with mechanical simplicity and low maintenance costs [3]. The parameters of the electrostatic demulsification process are voltage, frequency, temperature, degree of mixing, emulsion shape, and distance of electrodes, density and viscosity of emulsion, interfacial tension, water drop size, size distribution, hold up, surfactant concentration in the oil phase, and electrolyte concentration in the continuous phase [4-9]. The electrostatic demulsification process is based on the coalescence and precipitation of water droplets.

Although the electrostatic method has a relatively wide application, the mechanism of electrical coalescence does not well understood because of the complexity of hydrodynamic and electrical phenomena of interfacial polarization $[8,10,11]$. The electric field acts only at the interface between droplets and the continuous phase rather than throughout the bulk of the phases [10]. The diameter of the internal droplets of emulsion has a great influence on both the critical electric field intensity and the demulsification rate [7].

In this study, the effect of electrical field in term. of frequency and voltage on demulsification of water-in-oil emulsion before and after dispersion using glass coated electrode in batch system will be discussed. In addition, the effect of emulsion preparation such as homogenizer speed, internal phase and surfactant concentrations on the stability of water-in-oil emulsion was also investigated.

\subsection{EXPERIMENTAL PROCEDURES AND MATERIALS}

Kerosene was obtained from Fluka Chemika and sulphuric acid from Quality Reagent Chemical.
These chemicals were used as received. The apparatus used were; agitator IKKA C-MAG, homogeniser Heidolph Silent Crusher $M$, and high voltage demulsifier ISO-TECH ISR622 Oscilloscope. An emulsion was prepared by emulsifying aqueous solutions (stripping phase) with formulated organic phase following the method below.

An equal volume of $5 \mathrm{ml}$ portions of organic solutions and an aqueous strip solution were stirred at $8000 \mathrm{rpm}$ using a homogeniser for 5 min to obtain a continuously stable emulsion system. The emulsion was cooled down to room temperature prior to the demulsification study. The emulsions were freshly prepared for each of the experiments. For the purpose of extraction process, the emulsion will be dispersed into the agitated vessel with the external solution (water) in a volume ratio of 1:5 at $500 \mathrm{rpm}$ stirring speed. The two-phase dispersion was stirred for 5 minutes then the samples were quickly introduced in a separation funnel. The emulsion was separated from the aqueous phase. Finally, the emulsion was demulsified using high voltage demulsifier and demulsification time was recorded.

The model of batch demulsifier was equipped with a glass-insulated electrode as shown in Figure 1. The inner glass cylinder was filled with emulsion and glass coated stainless steel rod was placed in the center of the glass cylinder to serve as the inner electrode. The wall thickness of the glass coated was $1 \mathrm{~mm}$. The diameter of stainless steel rod was $10 \mathrm{~mm}$.

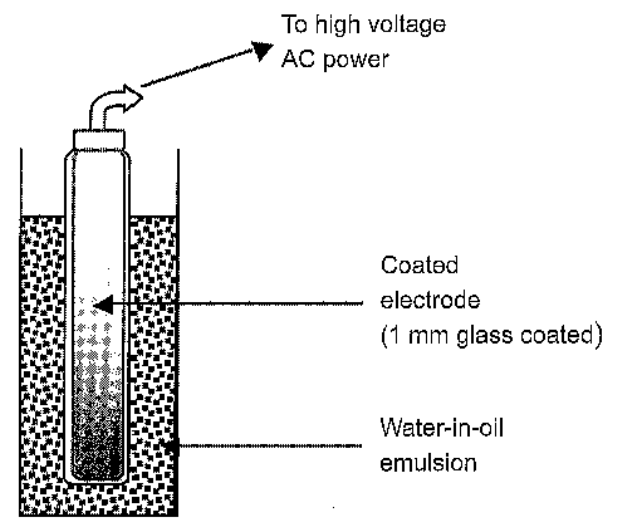

Figure 1 Schematic diagram of glass coated electrode in batch system 
Demulsification experiments were performed by applying a pulsed AC field with electric potential up to $25 \mathrm{kV}$ and $4000 \mathrm{~Hz}$ across the outer and inner electrode. The specification of high voltage demulsifier is shown in Table 1.

\subsection{RESULTS AND DISCUSSIONS}

\subsection{Effect of Applied Voltage}

The effect of applied voltage on demulsification of water-in-oil emulsion system of before and after dispersion is shown in Figure 2. The time required to breakdown the emulsion decreases when the applied voltage increased. This is due to under electrical field; the induced electrostatic force between two conducting drops is inversely proportional to approximately the fourth power of the separation distance between the drops [3]. Therefore, based on the van der Waals force, the electric induced force increases dramatically with decreasing separation between the drops and becomes important only when the drops are close to each other. However, this electric-induced

Table 1 Specification of high voltage demulsifier

\begin{tabular}{|c|c|c|c|}
\hline & $\begin{array}{l}\text { Power } \\
\text { Supply }\end{array}$ & $\begin{array}{l}\text { High Voltage } \\
\text { Power Supply }\end{array}$ & Accessories \\
\hline Input & $: 240 \mathrm{~V}$ & $: 0-22 \mathrm{~V}$ & Oscilloscope \\
\hline Output & $: 0-22 \mathrm{~V}$ & $: 0-25 \mathrm{kV}$ & High voltage probe \\
\hline Current & : 6 Amp & Frequency : $30-4000 \mathrm{~Hz}$ & \\
\hline \multicolumn{3}{|c|}{ Frequency: $60 \mathrm{~Hz}$} & \\
\hline
\end{tabular}

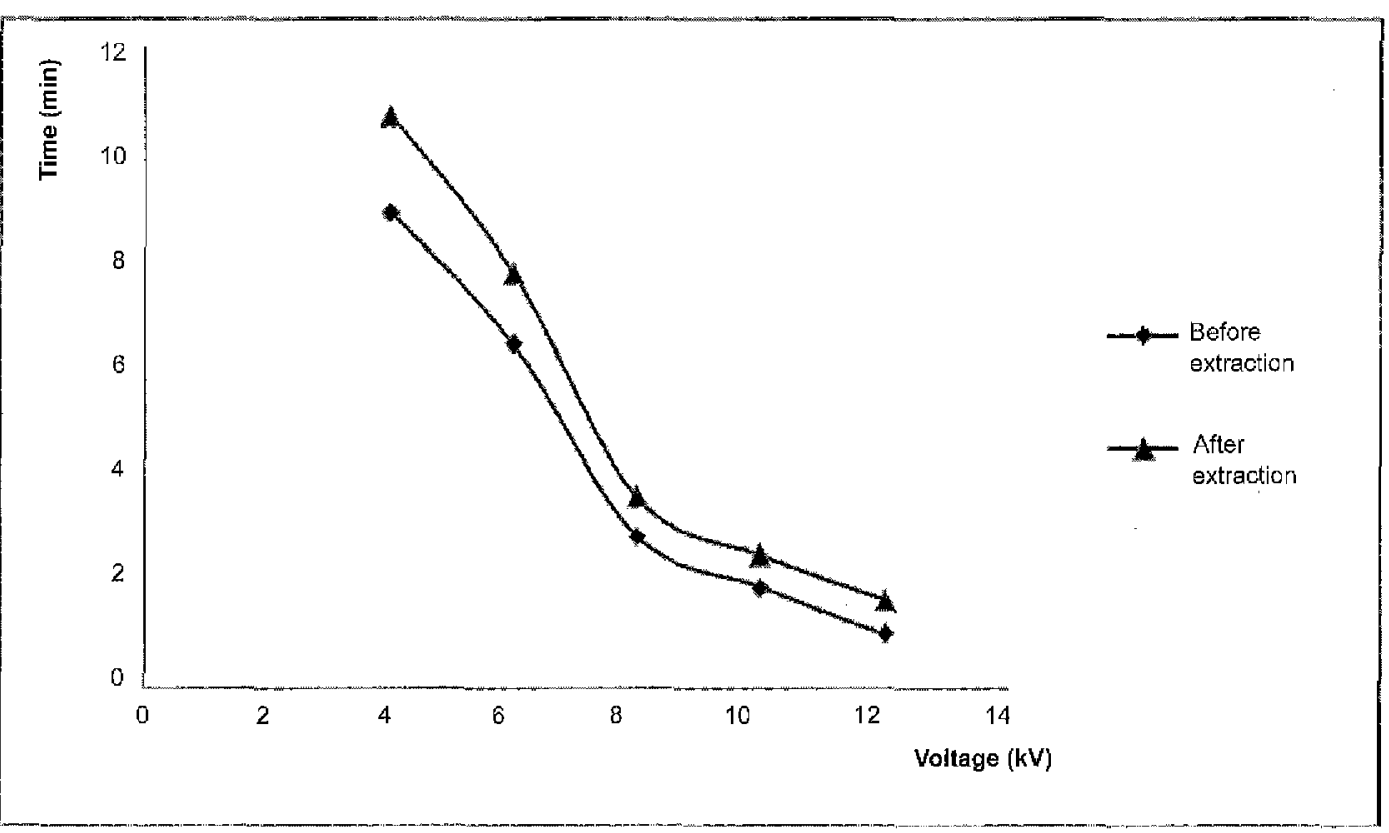

Figure 2 Demulsification of water-in-oil emulsion under various applied voltage (Experimental conditions: Span $80=5 \% \mathrm{w} / \mathrm{v}$, oil phase $=$ kerosene, water phase $=1 \mathrm{M}$ sulphuric acid, emulsifications speed $=8000 \mathrm{rpm}$, frequency $=500 \mathrm{~Hz}$, temperature $=27^{\circ} \mathrm{C}$, agitation speed $=500 \mathrm{rpm}$, agitation time $=5$ minutes) 
force also increases with the square of the drop size, thus dominating the van der Waals force in the coalescence of large drops. However, the field within the drop is not important with regard to coalescence. The coalescence rate improves as the applied electric field strength is increased [3] .

The mechanism of the electrostatic coalescence involved three-stage process. In the first stage, the drops approach each other and are separated by a film of the continuous phase. The second stage involves the thinning of the film to reduce the interfacial area and the last stage is the film rupture leading to droplet-droplet coalescence.

According to the mechanism of electrostatic coalescence, raising the applied electric field strength will increase the mutual attractive force between the water droplets in the electrical field, so accelerate the coalescence process [12]. With insulated electrode, coalescence efficiency increases with field strength [13]. Figure 2 also shows that time required for demulsification of W/O emulsion after dispersion is more than before dispersion due to Span 80 as a non-ionic surfactant or hydrophobic surfactant. Hydrophobic surfactants have a stabilizing effect on the double emulsion while the hydrophilic surfactants have a destabilizing effect [3].

\subsection{Effect of Frequency}

The time required for separation or breal down of water-in-oil emulsion as a function of frequency applied at constant voltage is presented in Table 2. The table shows the separation time decreases as the frequency increases for both emulsions of before and after dispersion.

This phenomenon indicates that under constant voltage condition, the demulsification rate increased with an increase in frequency. On the other hand, it can be explained that under highvoltage electrical field, the equivalent circuit of the coalescer containing an emulsion to be broken could be simplified to a resistor-capacitor series model, in which the resistance comes from the chains of water droplets in the water-in-oil emulsion and the insulation layer of the electrode could be regarded as a capacitor [14].

The table also shows that at low-frequency region, the time required for completely charging
Table 2 Demulsification of water-in-oil emulsion before and after dispersion (Span 80:5\% $\mathrm{w} / \mathrm{v}$, oil phase: kerosene, water phase: 1 M Sulphuric acid, emulsifications speed: $8000 \mathrm{rpm}$, voltage: $8 \mathrm{kV}$, emulsification time: 5 minutes, temperature: $27^{\circ} \mathrm{C}$ )

\begin{tabular}{ccc}
\hline $\begin{array}{c}\text { Frequency, } \\
\mathbf{f}(\mathbf{H z})\end{array}$ & $\begin{array}{c}\text { Before dispersion, } \\
\text { Time, } \mathbf{t} \text { (min) }\end{array}$ & $\begin{array}{c}\text { After dispersion, } \\
\text { Time, } \mathbf{t} \text { (min) }\end{array}$ \\
\hline 400 & 3.77 & 5.31 \\
500 & 2.68 & 3.58 \\
600 & 1.74 & 2.90 \\
700 & 1.09 & 2.16 \\
800 & 0.85 & 1.92 \\
1000 & 0.78 & 1.85 \\
1200 & 0.69 & 1.30 \\
1500 & 0.61 & 1.26 \\
\hline
\end{tabular}

and discharging the capacitor was sufficiently long. At frequency of $400 \mathrm{~Hz}$ the time required for before and after dispersion are 3.77 and 5.31 minutes, respectively. While at high frequency of $1500 \mathrm{~Hz}$ the time required for before and after dispersion are only 0.61 and 1.26 minutes, respectively. This result indicates that each pulse would cause one step of collision of water droplet. Therefore, raising the frequency would increase the collision number per unit time, thus enhancing the coalescence rate. However, as the frequency reached its critical value, the further increase in frequency would cause the incomplete charging of the capacitor thus will decrease the force acting on water droplets, resulting in a reduction of the demulsification rate [14].

\subsection{Effect of Surfactant Concentration}

Figure 3 shows the effect of applied voltage on the demulsification time at different concentration of Span 80 . The time required for water-in-oil emulsion to separate decreased as the applied voltage increases. However, at certain voltage the time required to emulsion breakdown depend on the concentration of Span 80 especially at low applied voltage. These trends are same for the both process, before and after dispersion that the emulsion stability increased with increasing 


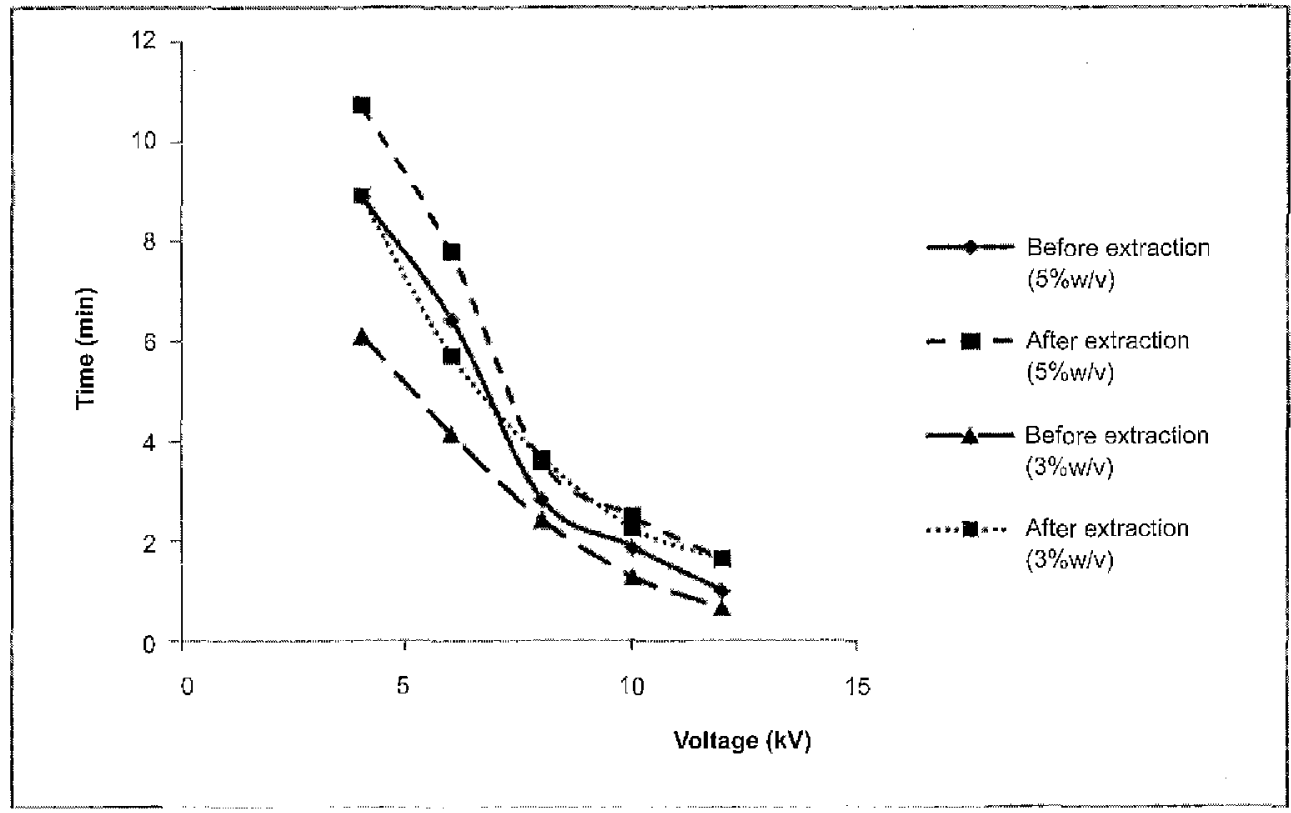

Figure 3 Demulsification of water-in-oil enulsion (oil phase: kerosene, water phase: 1 M Sulphuric acid, emulsifications speed: $8000 \mathrm{rpm}$, frequency $=500 \mathrm{~Hz}$, emulsification time: 5 minutes, temperature: $27^{\circ} \mathrm{C}$, stirring speed: $500 \mathrm{rpm}$, stirring time: 5 minutes)

surfactant concentration. The similar results have also found by Othman et al. [15].

At low concentrations of Span $80(3 \% \mathrm{w} / \mathrm{v})$, rapid coalescence among the inner and multiple droplets as well as loss of inner droplets to the external aqueous phase occurred, resulting in phase separation within a short period of time. Jiao and Burgess [16] have reported a similar result that the very fine inner aqueous droplets will be formed in increasing of surfactant concentration. Therefore, the results can be expressed that the inner aqueous droplets at $5 \%(\mathrm{w} / \mathrm{v})$ of Span 80 were smaller compared to $3 \%(w / v)$ of Span 80. The smaller initial droplet size provides an enhanced kinetic stability of the inner droplets to coalescence. The size of the inner droplets is estimated microscopically to be less than a micron [15]. The multiple droplets become smaller and their coalescence occurred less frequently with the formulations of $5 \%(\mathrm{w} / \mathrm{v})$ Span 80 . They concluded that increasing the concentration of surfactant will increase the stability of emulsion.

The interfacial tension between the membrane phase and the external phase decreases with increase in the surfactant (Span 80), whereas the viscosity of the membrane phase increases [17]. The membrane with high viscosity diluents is strong. Although this kind of membrane offers a lower extraction rate, it is more stable and thus has better overall extraction efficiency. An increase in surfactant concentration increases the viscosity and the stability of the adsorption layer, which in turn enhances resistance of the emulsion to mass transfer and invites more water transfer [12].

\subsection{Effect of Homogenizer Speed}

Figure 4 shows the effect of homogenizer speed on the demulsification time of water-in-oil emulsion. The results show that, the time required to breakdown the emulsion increase with increasing of homogenizer speed. This phenomenon indicated that high emulsification speed caused efficient emulsification and gives a good dispersion of internal phase drops into the membrane. Increasing emulsification speed produces smaller drops thus enhanced the surface area and talzes much more time to coalesce. The increase of emulsification speed will increase the emulsion stability. 


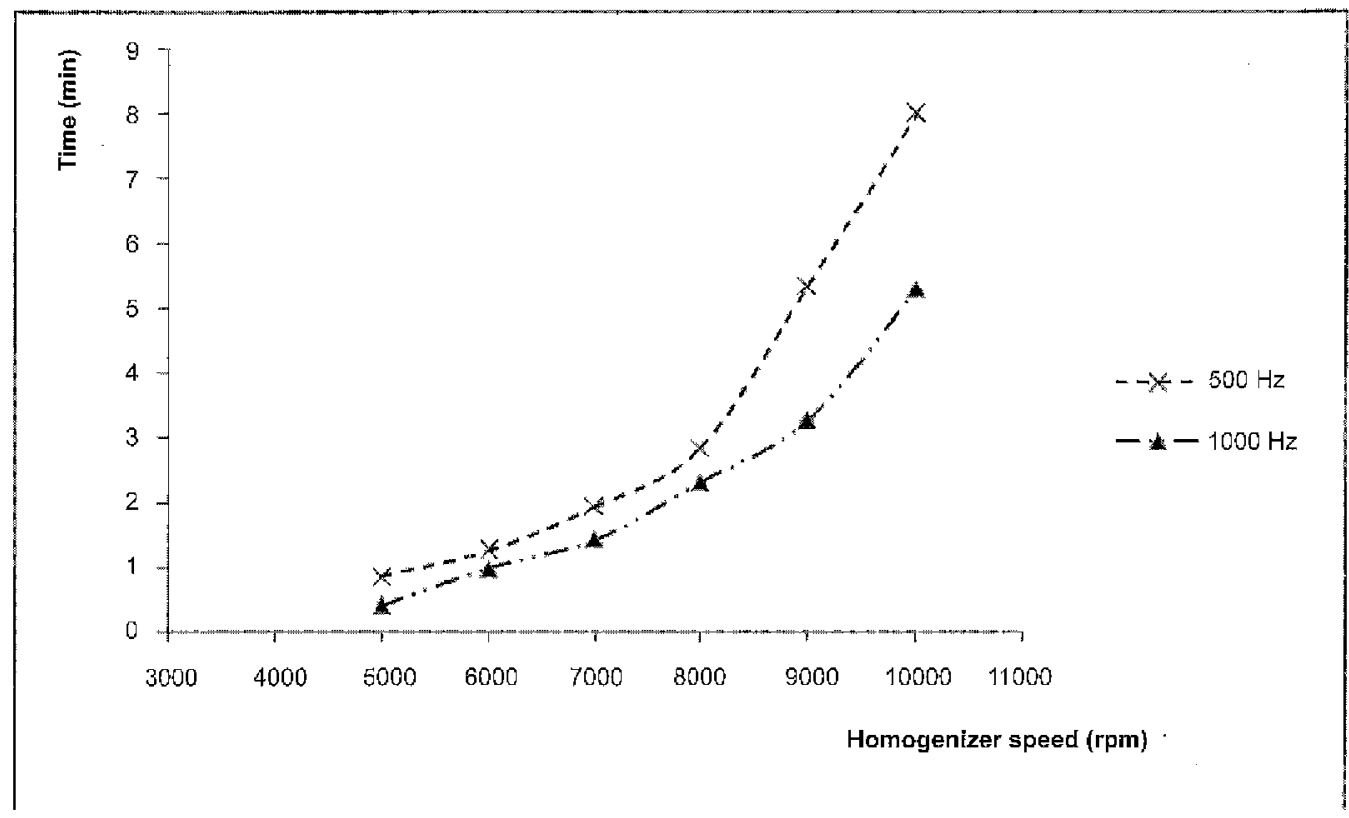

Figure 4 The demulsification of water-in-oil emulsion after dispersion (Span 80:5\% w/v, oil phase: kerosene, water phase: $1 \mathrm{M}$ sulphuric acid, emulsification time: 5 minutes, Applied voltage: $8 \mathrm{kV}$, temperature: $27^{\circ} \mathrm{C}$ )

This finding is in line as reported by Lissant [18] which indicates that the larger the particle, the less distance travels in any direction due to Brownian effects whereas snall particles have Brownian motion to deflect their already slower settling. His result indeed showed that larger particles settle quickly but smaller particles, due to Brownian displacement, take much longer to settle. It is remarkable when the emulsion drops become smaller, they will take much more time to coalesce. Chiha et al. [19] and Othman [15] also found the emulsion breakage decrease with the increase of the emulsification speed. An efficient emulsification gives a good dispersion as the internal phase drops into the membrane phase. These drops will become smaller and take much more time to coalesce.

The increasing the frequency of electrical field will be decreased the demulsification time. It means that increasing electrical strength will enhance the coalescence process. At low frequency, long chains are formed between the electrodes. Yan et al. [14] reported that under constant voltage, the critical (optimum) frequency existed. Below the critical frequency the demulsification tate was enhanced with increasing frequency, but above the critical it will decline with increasing frequency.

\subsection{CONCLUSIONS}

The increasing electrical field either applied voltage or frequency will decrease the separation time of water-in-oil emulsion for both before and after dispersion in emulsion liquid membrane process. The increasing electrical strength will enhance the coalescence process. At low frequency, long chains are formed between the electrodes.

Therefore, the effect of enulsion preparation such as homogenizer speeds and surfactant concentration affect the stability of emulsion and the time required for emulsion demulsification. The high emulsification speed caused efficient emulsification and gives a good dispersion of internal phase drops into the membrane. Increasing emulsification speed produces smaller drops thus enhanced the surface area and takes much more 
time to coalesce. The increase of emulsification speed will increase the emulsion stability. The affect of Brownian motion deflect also the slower settling of particles.

\section{ACKNOWLEDGEMENTS}

The authors would like to acknowledge the financial support from the Ministry of Higher Education (MOHE), Malaysia (FRGS No. 78042) and Universiti Teknologi Malaysia for making this study possible.

\section{REFERENCES}

[1] Shinoda, K. and S. Friberg. 1986. Emulsions and Solubilization. New York: John Wiley \& Sons.

[2] Eow, J. S. and G. Mojtaba. 2002. Electrostatic Enhancement of Coalescence of Water Droplets in Oil: A Review of the Technology. Chem. Eng. J. 85: 357-368.

[3] Eow, J. S., G. Mojtaba, A.O. Sharif, and T. J. Williams. 2001. Electrostatic Enhancement of Coalescence of Water Droplets in oil: A Review of the Current Understanding. Chem. Eng. J: 173-192.

[4] Ibrahim, M. Y., R. Mostafa, M. F. M. Fahmy, and A. I. Hafez. 2001. Utilization of Electroflotation in Remediation of Oily Wastewater. Sep. Sci. Technol. 36: 37493762 .

[5] Ivanov, I. B. and P. A. Kralchevsky, 1997. Stability of Emulsion Under Equilibrium and Dynamic Conditions. Colloids and Surfaces. 128: 155-175.

[6] Kim, B., J. H. Moon, S. Yang, and J. Kim, 2002. Demulsification of Water-in-Crude oil Emulsions by a Continuous Electrostatic Dehydrator. Sep. Sci. Technol. 37: 13071320.

[7] Lu, G., Q. Lu, and P. Li, 1997. Break-down of Liquid Membrane Emulsion under High Electric Field. J. Membr. Sci. 128: 1-6.

[8] Strathman, H. 2001. Membrane Separation Processes: Current Relevance and Future Opportunities. AlChE Journal, 47: 10771087.
[9] Wang, S.S., C. J. Lee, and C. C. Chan, 1994. Demulsification of Water-in-Oil Emulsions by Use of a High Voltage A. C. Field. Sep. Sci. Technol. 29: 159-170.

[10] Benavente, J. and G. Jonsson. 2000. Electro Kinetic Characterization of Composite Membranes: Estimation of Different Electrical Contributions in Pressure Induced Potential Measured Across Reverse Osmosis Membrane. J. Membr. Sci. 172: 189-197.

[11] Luo, G. S., S. Pan, J. G. Liu, and Y. Y. Dai. 2001. Liquid-liquid Phase Equilibrium under External Electric Fields. Sep. Sci. Technol. 36: 2799-2809.

[12] Ho, W. S. W. and K. K. Sirkar. 1992. Membrane Handbook. Second Edition. Fifth Aveneu, N. Y.: Van Nostrand Reinhold.

[13] Hauertmann, H., W. Degener, and $\mathrm{K}$. Schuger:. 1989. Electrostatic Coalescence: Reactor Process, Process Control, and Important parameters. Sep. Sci. Technol. 24: 253-273.

[14] Yan, Z., S.Y. Li, L.C. Wang, and F. Y. Yang. 1990. Demulsification of Liquid Membranes in Electric Fields. Journal of Water Treatment. 5: 1-6.

[15] Othman, N. 2006. Selective Emulsion Liquid Membrane Extraction of Silver from Liquid Photographic wastes. PhD Thesis (2006) Universiti Teknologi Malaysia.

[16] Jiao, J. and D. J. Burgess. 2003. Rheology and Stability of Water-in-oil-in-water Multiple Emulsions Containing Span 83 and Tween 80. Article of AAPS PharmSci. 5(1): Article 7.

[17] Yan, J. and R. Pal. 2003. Isotonic Selling Behavior of W/O/W Emulsion Liquid Membranes under Agitation Conditions. J. Membr. Sci. 213: 1-12.

[18] Lissant, K. J. 1983. Demulsification: Industrial Application. New York: Marcel Deliker, Inc.

[19] Chiha, M., M. H. Samar, and O. Hamdaoui. 2006. Extraction of Chromium (VI) from Sulphuric Acid Aqueous Solutions by a Liquid Surfactant Membrane (LSM). Desalination. 194: 69-80. 